\title{
Management of Bone Metastases From Breast Cancer in Upper and Lower Body at the Same Time: A Case Report
}

\author{
Gian Franco Ciaglia ${ }^{\mathrm{a}}$, Antonia Martino ${ }^{\mathrm{a}}$, Khadija Sayad ${ }^{\mathrm{a}}$, Claudio Scoglio ${ }^{\mathrm{a}}$, Donato Pezzulla ${ }^{\mathrm{a}, \mathrm{b}}$, \\ Salvatore Cappabianca ${ }^{\mathrm{a}}$
}

\begin{abstract}
Breast cancer is by far the most common cancer in women, and it has the highest incidence rates in western Europe. At breast cancer diagnosis, approximately $5-6 \%$ of women present with distant spread with bone, representing the most common site of metastatic lesions. More than half of the women, who present with metastatic breast cancer at the primary diagnosis, will develop bone metastases. We report a clinical case of a 75-year-old woman, with a history of breast cancer who undergone surgery 7 years ago, presenting bone metastases in different areas. We tried to determinate the major areas of pain and then to quantify it with a one-dimensional scale. After that, we analyzed the images of the previous instrumental exams and the centering CT in order to compare them with what the patient reported and then to decide what we should have targeted first. The aim of our work was to try finding a methodology in order to determinate the priority in the selecting of the area to treat to apply in this kind of patients.
\end{abstract}

Keywords: Metastases; Breast cancer; Multiple sites; Radiotherapy; Palliation

\section{Introduction}

Breast cancer is by far the most common cancer in women, and it has the highest incidence rates in western Europe [1]. At breast cancer diagnosis, approximately $5-6 \%$ of women present with distant spread with bone, representing the most common site of metastatic lesions [2,3]. More than half of the women, who present with metastatic breast cancer at the primary diagnosis, will develop bone metastases [4]. Breast cancer patients with bone metastases are at risk of skeletalrelated events including bone pain which requires palliative

\section{Manuscript accepted for publication March 29, 2016}

aDepartment of Internal Medicine, Clinical and Experimental "Magrassi-Lanzara", Section of Radiation Oncology, Seconda Universita di Napoli, Neaples, Italy

${ }^{\mathrm{b}}$ Corresponding Author: Donato Pezzulla, Corso Vittorio Emanuele 432, 80135, Neaples, Italy. Email: pezzulla.donato@libero.it

doi: http://dx.doi.org/10.14740/wjon966e radiotherapy. Radiation therapy is the most efficacious treatment for pain control in uncomplicated bone metastases [5].

\section{Case Report}

We report a clinical case of a 75-year-old woman, with a history of breast cancer who undergone surgery 7 years ago, presenting bone metastases in different areas. The lesion was found by a routine mammography 7 years ago and she was operated few months after the discovery. After 7 years of clinical silence from the diagnosis, the patient was submitted to a positron emission computed tomography (PET/CT) that showed multiple predominantly lytic lesions in several skeletal segments, especially to the left iliac wing and ipsilateral acetabulum, at the level of the right scapula and L4 and D10 vertebra. This result led to a palliative radiant treatment at the level of the L4 vertebrae with a total dose of $30 \mathrm{~Gy}$ in 10 fractions (3 $\mathrm{Gy} /$ fraction) provided with a $6 \mathrm{MeV}$ flash technique. After 2 months from the treatment, a total body CT was performed and it confirmed the previous lesions seen in the PET/CT and showed new lesions to the vertebra from D1 to D5. A further CT few months later pointed out an unchanged condition but there was also a worsening of the lytic lesions in D3 and D4, confirmed by an MRI 1 month later (Fig. 1). In February 2016, this woman arrived at our structure for a palliative treatment for the pain she felt at the level of her back and right scapula. We visited the patient and evaluated the pain level in the different sites through a numeric scale of evaluation (Karnofsky scheme) and an objective examination. After evaluating her clinical conditions and viewing the radiological exams, we decided to treat firstly the D2-D6 vertebra with a total dose of 30 Gy in 10 fractions ( $3 \mathrm{~Gy} /$ fraction) and then the scapula with a singular dose of 8 Gy provided with a 6 MV flash technique, where the patients felt the stronger pain, and not to treat the other lesions in areas where the pain was minimal or not existent. This way we save the opportunity to treat them when the patient will become symptomatic.

\section{Discussion}

Palliative radiotherapy (RT) is a non-invasive, highly effective and easily delivered treatment option for patients with meta- 


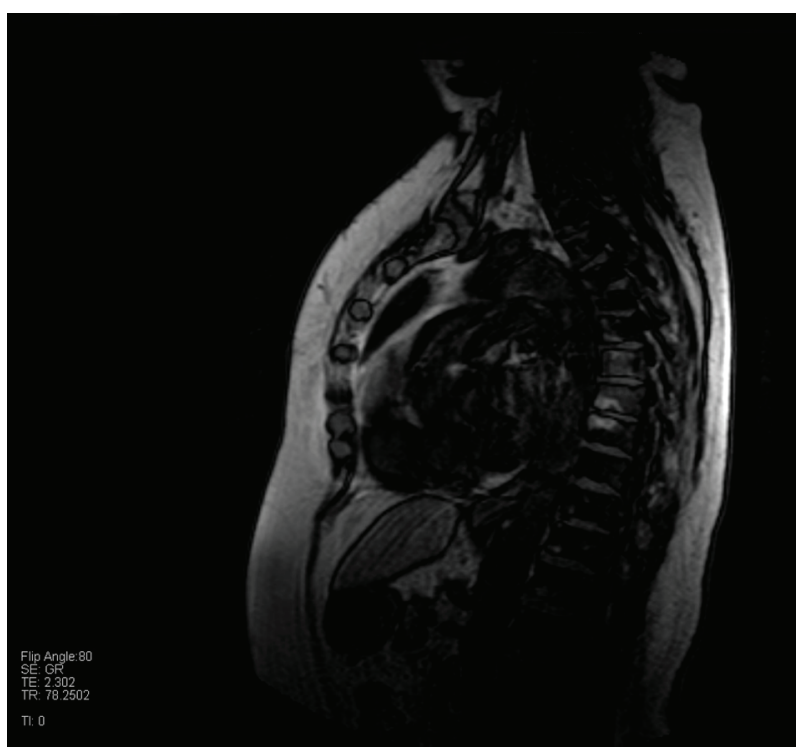

Figure 1. MRI shows signal alterations at the level of vertebrae from D1 to D5.

static bone disease. The main aim of RT is to prevent SREs and to palliate pain. Although the analgesic mechanism of RT is not fully understood, $75-85 \%$ of patients who received RT have a complete response on pain control discontinuing painkillers [6]. Although the total dose of RT administered depends on tumor radio-sensitivity and tolerance of tissues affected by the radiant beam, conventional fractionation involving daily fractions of 1.8 - 2 Gy (5 fractions per week) is generally used for the majority of bone lesions. Different fractionation schedules have also been evaluated in these years: hyperfractionated RT, in which the total dose of radiation is divided into small doses and treatments are given more than once a day; hypofractionated RT, in which the total dose of radiation is divided into large doses and treatments are given once a day or less often [7].

In this case, we have a patient with lesions both in the upper and lower part of her body, so we had to decide the priority in the choosing the target and the timing in treating them. What guided us to the selection of the target was at first the colloquium with the woman and the objective examination: we tried to determinate the major areas of pain and then to quantify it with a one-dimensional scale. After that, we analyzed the im- ages of the previous instrumental exams and the centering CT in order to compare them with what the patient reported and then to decide what we should have targeted first.

At the moment in literature there are only few works relating to the metastases management in this kind of situation and there are not official radiotherapic guidelines we could use to treat these patients.

\section{Conflicts of Interest}

There is no conflict of interest.

\section{References}

1. Ferlay J, Shin HR, Bray F, Forman D, Mathers 1. C, Parkin DM:GLOBOCAN 2008 v1.2, Cancer Incidence and Mortality Worldwide: IARC CancerBase No. 10 [Internet]. Lyon, France: International Agency for Research on Cancer; 2010. Available from http://globocan.iarc.fr. Accessed May 2011.

2. Sogaard KK, Cronin-Fenton DP, Pedersen L, Sorensen HT, Lash TL. Survival in Danish patients with breast cancer and inflammatory bowel disease: a nationwide cohort study. Inflamm Bowel Dis. 2008;14(4):519-525.

3. Louwman WJ, Voogd AC, van Dijck JA, Nieuwenhuijzen GA, Ribot J, Pruijt JF, Coebergh JW. On the rising trends of incidence and prognosis for breast cancer patients diagnosed 1975-2004: a long-term population-based study in southeastern Netherlands. Cancer Causes Control. 2008;19(1):97-106.

4. Coleman RE, Rubens RD. The clinical course of bone metastases from breast cancer. Br J Cancer. 1987;55(1):6166.

5. Maisano R, Pergolizzi S, Cascinu S. Novel therapeutic approaches to cancer patients with bone metastasis. Crit Rev Oncol Hematol. 2001;40(3):239-250.

6. Fontanella C, Fanotto V, Rihawi K, Aprile G, Puglisi F. Skeletal metastases from breast cancer: pathogenesis of bone tropism and treatment strategy. Clin Exp Metastasis. 2015;32(8):819-833.

7. Beyzadeoglu M, Ozyigit G, Ebruli C. Basic radiation oncology. Springer, Heidelberg, 2010. 